\title{
Conceptualizing the intersubjective group
}

\author{
Gerry Stahl ${ }^{1}$
}

Published online: 13 August 2015

(C) International Society of the Learning Sciences, Inc. 2015

Intersubjectivity may be considered the defining characteristic of CSCL. Intersubjectivity is a concept that indicates shared understanding among people. This "sharing" is not a matter of individuals having similar understandings, but of them participating productively in a joint meaning-making discourse within a communal world. A group has achieved intersubjectivity if the members of the group interact well enough to pursue the group's aims. Intersubjectivity must be built up gradually through interaction and repaired frequently. CSCL research explores the conditions and processes that are conducive to the establishment and maintenance of intersubjectivity among groups of learners. CSCL pedagogies promote the intersubjectively shared understanding that makes collaborative learning possible. CSCL technologies support intersubjectivity by providing media of communication and scaffolds for meaning making within a specific domain of learning.

When CSCL theories discuss "groups," they are not referring to arbitrary gatherings of multiple learners, but to functional groups that have achieved a degree of intersubjectivity. The concept of collaborative learning in CSCL does not refer to a sum of individual learning that takes place among a group's members, but to the increase in intersubjective understanding or knowledge building within the group that results from joint meaning making in a shared context. It involves the understanding expressed in the group discourse and the knowledge encapsulated in group products, texts or artifacts. The group's understanding may differ from what any individual member might say, write or think when not interacting within the group.

This focus on the intersubjective group differentiates CSCL from other approaches to the study of human learning and educational instruction. It implies a research paradigm that prioritizes the group unit of analysis and studies groups that have achieved intersubjectivity. Analyzing an utterance (or chat posting) as part of a group interaction involves seeing how its meaning is constructed sequentially through its response to previous actions and elicitation of future behavior by other group members. The meaning of the utterance is inherent in the working of that utterance within the shared world of the group, not to be explained in terms of some purported individual mental thoughts accompanying the utterance. As in Ryle's (1968)

Gerry Stahl

Gerry@ijCSCL.org 
thick description of a wink, the meaning of a wink (or utterance) is expressed by the wink itself as an interactional action, not by assumed additional intentions of the winker.

Despite the centrality of the notion of intersubjectivity to CSCL, this concept has not often been explicitly discussed in the CSCL literature. Newcomers to CSCL therefore have difficulty determining the boundaries of the field. They may assume that CSCL is the same as traditional educational psychology or instructional design, except that it involves small groups and online technology. However, the importance of analyzing intersubjectivity at the group unit of analysis has become increasingly clear to many established CSCL practitioners. For instance, the $i j C S C L$ Mission Statement now specifies that the journal "features empirically grounded studies and descriptive analyses of interaction in groups, which investigate the emergence, development and use of practices, processes and mechanisms of collaborative learning." The central research questions are no longer what experimental conditions produce the most valued learning experiences or outcomes at the individual unit, but how intersubjective meaning making and understanding is established, maintained and increased within the interaction in groups, by social practices, small-group processes and interactional mechanisms analyzed at the group unit.

The shift of research from assessing individual student outcomes to analyzing group-level phenomena has been slow coming and is still difficult to implement consistently. In the late 1900s, educational researchers like Johnson and Johnson (1999) or Slavin (1980) explored the effects of group interaction on learning outcomes of individual students; this was called cooperative learning. With the advent of CSCL, interest changed to the group processes that could be supported with networked-computer technologies. In their report on the evolution of research on collaborative learning, Dillenbourg et al. (1996) noted that new methods were now necessary to study group phenomena. Although Koschmann (1996) proposed that this involved a paradigm shift, it has not been widely recognized what a radical change in perspective and methodology this shift to the group level implied.

Subsequently, Koschmann (2002) defined CSCL in terms of "joint meaning making"- the focus of the opening article in this issue. The centrality of intersubjective meaning making to the concerns of CSCL as a research field have been stressed programmatically in scattered proposals and examples, for instance in (Arnseth and Ludvigsen 2006; Çakir et al. 2009; Suthers 2006; Suthers et al. 2010). Multiple attempts to define new methods corresponding to this agenda of group-level analysis were also proposed, as in several ijCSCL articles (Cress 2008; Cress and Kimmerle 2008; Damsa 2014; Furberg et al. 2013; Noroozi et al. 2013; Öner 2013; Overdijk et al. 2014; Zemel and Koschmann 2013; Zhao and Chan 2014).

After 20 years, CSCL researchers are just beginning to work out group-level conceptualizations, such as group cognition, group knowledge construction, group agency, group engagement, group metacognition, group practices and so on. Some researchers now see CSCL as pursuing a post-cognitive paradigm distinguished from the cognitivism of the learning sciences (Stahl 2015). For instance, I have recently been exploring this post-cognitive paradigm through a theory of group cognition (Stahl 2014), a review of philosophical analyses of intersubjectivity (Stahl 2016b), design-based research on software support for multi-user problem solving (Stahl 2013) and a longitudinal study of group-cognitive development (Stahl 2016a). Such a post-cognitive approach may distinguish CSCL most clearly from the methodological individualism of the educational psychology, artificial intelligence and learning sciences from which it emerged.

The current issue of $i j C S C L$ provides a set of stimulating papers that illustrate and further develop a group-level focus of CSCL research. First, a discussion of Habermas' philosophy as 
it relates to CSCL issues introduces to the CSCL audience the work of the contemporary author who has written the most on the concept of intersubjectivity. Then, three papers analyze the intersubjectivity of small groups of students in different ways. One looks at how groups learn how to learn together with support from specific CSCL tools. A second transforms the concept of engagement to the group unit of analysis as collaborative group engagement. The final one makes a parallel move for formative feedback and metadiscourse, applying them at the group level. Together, they offer stimulating glimpses of CSCL theory, technology, metalearning and analysis focused on the group as agent.

Of course, the emphasis on group-level intersubjectivity defines just one of the paradigms active in the CSCL research community. Certainly, not all CSCL researchers identify with a post-cognitive paradigm. Perhaps the much-debated distinction between quantitative and qualitative methods should be replaced with consideration of the unit of analysis as contrasting different CSCL paradigms. CSCL has always incorporated a diversity of methodological perspectives, and ijCSCL has always published leading statements from all the different approaches. While this issue emphasizes studies at the group unit of analysis, future issues will continue to highlight studies of individual outcomes or community participation.

\section{The conditions of the possibility of intersubjectivity}

In his introduction of Habermas' philosophy of communicative action to the CSCL community, Michael Hammond translates from Habermas' application of this theory in the public sphere of traditional media to the online world of CSCL. For him, Habermas is relevant because he brings a fresh, well-considered and critical perspective to the discussion of joint knowledge building. In particular, Habermas' writings provide a framework for judging the evidence we bring to the analysis of collaborative learning as well as for valuing the evidence that our student subjects provide in their argumentation. Habermas defines the conditions necessary for the establishment of intersubjectivity, such as the inherent assumption of an ideal speech situation underlying communicative action. What Kant's (1787/1999) Critique of Pure Reason did for the individual mind, articulating the conditions of the possibility of human knowledge, Habermas translated to the group level, explicating fundamental discourse conditions necessary for intersubjective meaning making in social collectivities.

Consider a student chat, a discussion forum or a medium like Wikipedia. How should we judge the quality of the knowledge building that takes place there? Moreover, how should one judge the quality of researchers' analysis of that knowledge building? Habermas provides a standard for judgment that is grounded in the nature of human discourse. He argues that effective communication would be impossible without the underlying postulation of an ideal speech situation — even if this ideal is never in fact achievable (Habermas 1981/1984). The act of communicating with the aim of establishing intersubjectivity, making shared meaning and building knowledge together assumes that there is no other force of persuasion at work than that of the better argument and no other motivation than the cooperative search for truth. Enlightened discourse is only possible under the assumption of this goal. Of course, there always are other forces and motivations present. But the character of the ideal speech situation that underlies collaborative dialog provides a basis for critiquing those systematically distorting forces. For instance, if knowledge building assumes that no one can impose his or her views through force rather than through supported reasoning, then appeals to authority or intimidation can be soundly censured. 
Habermas' theory is, additionally, more complex and nuanced. A major contribution of his work was to distinguish realms with different criteria within the public sphere (Habermas 1967/1971). There is, as Hammond puts it, the objective world (of nature and labor), the social world (of institutions and interaction) and the subjective world (of personal experience). Each has very different criteria of validity. The objective world follows the laws of physics and involves human mastery over nature through technical, goal-oriented, instrumental calculation; the social world, in contrast, involves normative rules reached through negotiation; while the subjective world is a matter of one's self-narrative.

Consider the research task of analyzing an online team of students collaborating on a geometry construction. Certainly, this involves comparing the team's work with mathematical knowledge developed in the objective world of mathematical relationships. However, it also involves tracking the development of the team's adoption and mastery of its own group practices of collaborating and of working on geometry in the team's social world. Furthermore, it may be possible to assess individual learning by team members as a personal-world spin-off of their teamwork. Each of these dimensions has quite different methodological criteria. Seeing how each is accomplished with the mediation of specific CSCL pedagogical approaches or CSCL technological tools can feed into design-based research for improving support for collaborative knowledge building.

Habermas' distinction between the objective, social and subjective realms gives him leverage for his critiques of modernism and other popular philosophies, extending the critical social theory of the Frankfurt School. As cited by Hammond, Habermas' concern with mutual recognition led him to criticize classical liberalism for reducing ethical liberty to a "possessiveindividualist reading of subjective rights, misunderstood in instrumentalist terms." There are many analogous examples in the CSCL literature, where social phenomena are inappropriately reduced either to individual subjective criteria or to instrumental objective criteria. Hammond suggests that a focus on intersubjectivity could provide a corrective in such cases and open up new perspectives for design and research. It is important to distinguish different levels of analysis carefully and to apply the appropriate evaluative criteria or analytic methods to each.

\section{Intersubjective learning to learn}

Teaching students to learn how to learn or to develop thinking skills has long been considered important (e.g., Looi et al. 2011; Wegerif 2006) - particularly in the information age, where knowledge evolves rapidly. In their research report, Baruch B. Schwarz, Reuma de Groot, Manolis Mavrikis and Toby Dragon extend this goal to the group level with their construct of learning-to-learn-together. A core component of this approach is supporting groups of students to engage in argumentation as a form of intersubjective meaning making. Schwarz and colleagues have previously published studies of CSCL support for argumentation in ijCSCL (Asterhan and Schwarz 2010; Schwarz and De Groot 2007; Schwarz and Glassner 2007; Schwarz et al. 2011; Slakmon and Schwarz 2014). Now they situate computer support for argumentation in an innovative dual-interaction space.

The authors take a design-based-research approach to developing a software environment, curricular tasks and teacher roles for supporting learning-to-learn-together. They hypothesize that mutual engagement, collective reflection and peer assessment may be three critical group processes to encourage and to investigate. To explore these, they design a prototype with two primary components: a construction space and an argumentation space. The construction space 
includes a selection of domain-specific modeling applications to support student inquiry in specific topics of mathematics or science. This provides a mutually visible "joint problem space" (Teasley and Roschelle 1993) for collective reflection by the group on the progress of its inquiry. The software creates a shared world for mutual engagement, as opposed to individuals trying to solve a challenging problem on their own. As one group member performs an action in the space, the others assess that action in the argumentation space, either affirming it or questioning it. This prompts the students to build on each other's actions, producing a joint accomplishment.

In some dual-interaction systems, a simple chat feature accompanies an online construction space (Lonchamp 2009; Mühlpfordt and Wessner 2009; Zemel and Koschmann 2013). This provides the possibility of engaged discourse, group reflection and peer assessment when group members are not situated face-to-face. However, the described Metafora system goes beyond this with a sophisticated planning/reflection tool. Even if the students are sitting together around a shared computer, this tool prompts, guides and supports team efforts at planning steps for the group to take (collective agency) and it facilitates team reflection on the current state (collective responsibility) (Scardamalia and Bereiter 2014). While the software mainly displays advice and ideas from the teacher or from individual students, its persistent visibility and its manipulable structure allow it to influence group agency and meta-learning. The potential power of this approach seems to come from the integration of the support for argumentation and reflection by the group with the inquiry activity itself in the shared inquiry environment. As always in CSCL, success also depends on a culture of collaboration: appropriate motivations/rewards, careful training in collaboration and subtle mentoring. The emphasis of the pedagogy and the support throughout is on the group as meta-learner.

\section{Intersubjective engagement}

In the next presentation, Suparna Sinha, Toni Kempler Rogat, Karlyn R. Adams-Wiggins and Cindy E. Hmelo-Silver provide a multi-faceted conceptualization and operationalization of intersubjectivity based on aspects of what they term "group engagement." Using this approach, they provide a clear illustration of a team of students that does not form an intersubjective group contrasted by one that does. The construct of group engagement developed in this paper allows the authors to identify this contrast and to analyze it using both quantitative and qualitative methods. The quantitative approach includes statistical correlations based on ratings of several aspects of group engagement, measured in five-minute intervals. The qualitative approach involves thick descriptions of illustrative excerpts of group discourse. The descriptions relate the interactions within the groups to their work (or lack thereof) of meaning making in establishing the engagement of the group as a whole in its problem-solving task.

A major achievement of the paper is to shift the analysis of engagement-which is increasingly popular in CSCL - from the psychological individual to the intersubjective group unit of analysis. The authors are explicit about this. Their observational protocol is designed to situate engagement within the collaborative group, its joint problem and its shared situation. For instance, the dimension of social engagement reflects group cohesion, or evidence that the task is conceptualized as a team effort, rather than as an individual activity. The contrast of one group's use of the subject "we" versus the other's use of "I" reflects in the details of the discourse the distinction documented in the ratings - showing that the distinction is actually one made by the group. 
The paper is an impressive response to the cited prior research on engagement. According to the literature review, earlier studies generally operationalized engagement as consisting of a single dimension, as a stable state and as a characteristic of the individual learner. In addition, the cited work decontextualized engagement from concomitant conceptual and disciplinary tasks. By contrast, this study proposes a differentiated, evolving, multi-faceted and groupbased model of engagement and applies this model to explore an insightful example from actual classroom practice. The paper's mixed-methods analysis reflects a careful attention to the unit of analysis, operationalizing engagement at the group level. Thereby, it adds in a rich way to our conceptualization of intersubjective meaning making.

\section{Intersubjective metadiscourse}

Like the preceding paper, the one by Monica Resendes, Marlene Scardamalia, Carl Bereiter, Bodong Chen and Cindy Halewood also uses mixed methods, with both quantitative and qualitative analysis. While collecting data at both the individual and group units of analysis, its focus is also at the group unit. In fact, it goes a step further than the previous paper and most other CSCL reports by capturing the outcomes at the group level. Here, because the main data source is a Knowledge Forum database, the group product of shared notes responding to each other within the group is the most important object for examination in response to the primary research question. Thereby, the correlation of the experimental condition with resultant collaborative learning or knowledge building can be conducted at the group level.

The social-network analysis of the Knowledge Forum notes shows the effect of experimental feedback tools on the group process and the degree of intersubjectivity established by each group. The striking visual contrast in the paper's Figure 5 indicates that in the control condition most students are not strongly connected to other students, whereas in the experimental condition everyone is strongly connected to everyone else. Because the connections here represent sharing of vocabulary terms - such as those displayed in the experimental condition's feedback tool - this means that there is a higher degree of intersubjective, shared understanding in the experimental groups. Shared understanding at the group unit of analysis is not dependent upon individuals' cognitive states, internal representations, or personal understandings, but is visibly displayed in the team's unproblematic use of shared language.

We are shown further evidence of increased group metadiscourse through the analysis of group discussion in a number of propitious interaction excerpts. While these demonstrate the experimental group's comprehension of the visualizations of their group discourse (displays of its use of domain vocabulary and of Knowledge Forum epistemic markers), the primary metadiscourse moves (prompting the group to plan, question, analyze, explain) were made by the teacher, rather than by the student group. The experimental intervention at the group level led to productive metadiscourse, but this was not at all independent of the teacher. Thus, the study merely indicates a potential for the design of formative assessment visualizations that represent group-level behaviors and that support group metadiscourse. It does not demonstrate that the implemented tools led to student metadiscourse on their own. The students may need more experience with this approach or more maturity to take on this form of agency within the student group. Nevertheless, the paper offers stimulating design suggestions: group-level formative feedback can represent group vocabulary; support the group to evaluate its own progress; give feedback on secondary processes (like vocabulary building, rather than directly on learning or task accomplishment); suggest positive steps (rather than just identify 
deficiencies); facilitate self-assessment by the group; and guide individual students to become more effective group members.

Together, the papers in this issue of $i j C S C L$ suggest the centrality of intersubjectivity to a theory of CSCL and provide inspiring examples of how to explore and articulate aspects of our conceptualization of group intersubjectivity.

\section{Report from CSCL 2015}

Just before traveling to Gothenburg, Sweden, for the CSCL conference, we were shocked to hear that Naomi Miyake had succumbed to a long illness. This was the first death of a member of the ijCSCL Editorial Board. Naomi was a founding member of the journal and had contributed reviews, advice and encouragement, even during crisis periods in Japan and with her own health. The conference began with a ceremony honoring Naomi. Comments from that event are included in the following memorial statement by Marcia C. Linn, Hajime Shirouzu and Masaki Miyake. At the end of the conference, the Naomi Miyake Best Student Paper Award was named in memory of Naomi from now on, in recognition of her commitment to promoting new research talent.

During the conference, ijCSCL held its annual Board meeting. We first expressed our deep sorrow that Naomi will no longer be with us except in spirit. Then the Board engaged in a lively discussion of the future of CSCL and how the journal can expand to support the growth of the field-globally, methodologically and in terms of content. It was pointed out that submissions to the journal are coming from more and more parts of the world. For instance, the journal has recently published several articles from Mainland China. Also, downloads of articles from the Springer $i j C S C L$ website are evenly divided among Asia, Europe and North America. However, some Board members were concerned that the scope of the journal is perceived as being too narrow to attract submissions from new approaches or from expanding areas of technology and methods. The Board determined to open the journal to new themes that have arisen around the periphery and potential future of the CSCL research field. In particular, the Board agreed to solicit articles in the following areas. Note that ijCSCL has already begun to discuss a number of these topics in the current and recent issues.

- Social Software,

- MOOCs,

- Tangibles,

- Mass Communication,

- Learning Analytics,

- Teacher Learning,

- Sensors,

- Vocational Ed \& Training,

- Informal/Civic/Lifelong Learning,

- CSCL@Work.

For such new areas, it might be ideal to start with an overview to introduce the recent literature and state of the art of the area to the ijCSCL readership. Then a couple papers could be published illustrating the relevance of the area to CSCL, along with a call for follow-up papers on the theme. This is $i j C S C L$ 's alternative to publishing special issues. 
The articles presented in the current issue illustrate this approach: the article on Habermas introduces the theme of intersubjectivity and its relevance to CSCL research, while the other papers provide studies of aspects of intersubjectivity in CSCL. If you are interested in submitting a paper or organizing a thread of related papers but are not sure whether it fits the expanded scope of ijCSCL, please contact an editor at exec@ijCSCL.org. A Board member might be willing to work with you to help frame your initiative.

\section{References}

Arnseth, H. C., \& Ludvigsen, S. (2006). Approaching institutional contexts: Systemic versus dialogic research in CSCL. International Journal of Computer-Supported Collaborative Learning, 1(2), 167-185.

Asterhan, C. S. C., \& Schwarz, B. B. (2010). Online moderation of synchronous e-argumentation. International Journal of Computer-Supported Collaborative Learning, 5(3), 259-282.

Çakir, M. P., Zemel, A., \& Stahl, G. (2009). The joint organization of interaction within a multimodal CSCL medium. International Journal of Computer-Supported Collaborative Learning, 4(2), 115-149.

Cress, U. (2008). The need for considering multilevel analysis in CSCL research: An appeal for the use of more advanced statistical methods. International Journal of Computer-Supported Collaborative Learning, 3(1), 69-84.

Cress, U., \& Kimmerle, J. (2008). A systemic and cognitive view on collaborative knowledge building with wikis. International Journal of Computer-Supported Collaborative Learning, 3(2), 105-122.

Damsa, C. I. (2014). The multi-layered nature of small-group learning: Productive interactions in object-oriented collaboration. International Journal of Computer-Supported Collaborative Learning, 9(3), 247-281.

Dillenbourg, P., Baker, M., Blaye, A., \& O’Malley, C. (1996). The evolution of research on collaborative learning. In P. Reimann \& H. Spada (Eds.), Learning in humans and machines: Towards an interdisciplinary learning science (pp. 189-211). Oxford: Elsevier. Web: http://tecfa.unige.ch/tecfa/publicat/dil-papers-2/Dil. 7.1.10.pdf.

Furberg, A., Kluge, A., \& Ludvigsen, S. (2013). Student sensemaking with science diagrams in a computerbased setting. International Journal of Computer-Supported Collaborative Learning, 8(1), 41-64.

Habermas, J. (1967/1971). Labor and interaction: Remarks on Hegel's Jena philosophy of mind. In Theory and practice. (pp. 142-169). Boston, MA: Beacon Press.

Habermas, J. (1981/1984). Reason and the rationalization of society (T. McCarthy, Trans. Vol. One). Boston, MA: Beacon Press. The theory of communicative action.

Johnson, D. W., \& Johnson, R. T. (1999). Making cooperative learning work. Theory Into Practice, 38, 67-73.

Kant, I. (1787/1999). Critique of pure reason. Cambridge, UK: Cambridge University Press.

Koschmann, T. (1996). Paradigm shifts and instructional technology. In T. Koschmann (Ed.), CSCL: Theory and practice of an emerging paradigm (pp. 1-23). Mahwah: Lawrence Erlbaum.

Koschmann, T. (2002). Dewey's contribution to the foundations of CSCL research. In G. Stahl (Ed.), Computer support for collaborative learning: Foundations for a CSCL community: Proceedings of CSCL 2002 (pp. 17-22). Boulder: Lawrence Erlbaum Associates.

Lonchamp, J. (2009). A three-level analysis of collaborative learning in dual-interaction spaces. International Journal of Computer-Supported Collaborative Learning, 4(3), 289-317.

Looi, C. K., So, H. J., Toh, Y., \& Chen, W. L. (2011). The Singapore experience: Synergy of national policy, classroom practice and design research. International Journal of Computer-Supported Collaborative Learning, 6(1), 9-37.

Mühlpfordt, M., \& Wessner, M. (2009). The integration of dual-interaction spaces. In G. Stahl (Ed.), Studying virtual math teams. (ch. 15, pp. 281-293). New York, NY: Springer.

Noroozi, O., Teasley, S. D., Biemans, H. J. A., Weinberger, A., \& Mulder, M. (2013). Facilitating learning in multidisciplinary groups with transactive CSCL scripts. International Journal of Computer-Supported Collaborative Learning, 8(2), 189-223.

Öner, D. (2013). Analyzing group coordination when solving geometry problems with dynamic geometry software. International Journal of Computer-Supported Collaborative Learning, 8(1), 13-39.

Overdijk, M., van Diggelen, W., Andriessen, J., \& Kirschner, P. A. (2014). How to bring a technical artifact into use: A micro-developmental perspective. International Journal of Computer-Supported Collaborative Learning, 9(3), 283-303.

Ryle, G. (1968). The thinking of thoughts: What is 'le penseur' doing? In University lectures, no.18. Canada: University of Saskatchewan. Web: http://lucy.ukc.ac.uk/CSACSIA/Vol14/Papers/ryle_1.html. 
Scardamalia, M., \& Bereiter, C. (2014). Knowledge building and knowledge creation: Theory, pedagogy and technology. In K. Sawyer (Ed.), Cambridge handbook of the learning sciences (2nd ed.). Cambridge: Cambridge University Press.

Schwarz, B. B., \& De Groot, R. (2007). Argumentation in a changing world. International Journal of ComputerSupported Collaborative Learning, 2(2-3), 297-313.

Schwarz, B. B., \& Glassner, A. (2007). The role of floor control and of ontology in argumentative activities with discussion-based tools. International Journal of Computer-Supported Collaborative Learning, 2(4), 449478.

Schwarz, B. B., Schur, Y., Pensso, H., \& Tayer, N. (2011). Perspective taking and synchronous argumentation for learning the day/night cycle. International Journal of Computer-Supported Collaborative Learning, 6(1), $113-138$

Slakmon, B., \& Schwarz, B. B. (2014). Disengaged students and dialogic learning: The role of CSCL affordances. International Journal of Computer-Supported Collaborative Learning, 9(2), 157-183.

Slavin, R. (1980). Cooperative learning. Review of Educational Research, 50(2), 315-342.

Stahl, G. (2013). Translating Euclid: Designing a human-centered mathematics. San Rafael, CA: Morgan \& Claypool Publishers. Web: http://GerryStahl.net/elibrary/euclid.

Stahl, G. (2014). The constitution of group cognition. In L. Shapiro (Ed.), Handbook of embodied cognition. New York: Routledge. Web: http://GerryStahl.net/pub/embodied.pdf.

Stahl, G. (2015). The group as paradigmatic unit of analysis: The contested relationship of CSCL to the learning sciences. In M. A. Evans, M. J. Packer, \& R. K. Sawyer (Eds.), Reflections on the learning sciences. New York: Cambridge University Press. Web: http://GerryStahl.net/pub/ls.pdf.

Stahl, G. (2016a). Constructing dynamic triangles together: The development of mathematical group cognition. Cambridge: Cambridge University Press. Web: http://GerryStahl.net/elibrary/analysis.

Stahl, G. (2016b). From intersubjectivity to group cognition. Computer Supported Cooperative Work. Web: http://GerryStahl.net/pub/intersubjectivity.pdf.

Suthers, D. D. (2006). Technology affordances for intersubjective meaning making: A research agenda for CSCL. International Journal of Computer-Supported Collaborative Learning, 1(3), 315-337.

Suthers, D. D., Dwyer, N., Medina, R., \& Vatrapu, R. (2010). A framework for conceptualizing, representing, and analyzing distributed interaction. International Journal of Computer-Supported Collaborative Learning, 5(1), 5-42.

Teasley, S. D., \& Roschelle, J. (1993). Constructing a joint problem space: The computer as a tool for sharing knowledge. In S. P. Lajoie \& S. J. Derry (Eds.), Computers as cognitive tools (pp. 229-258). Mahwah: Lawrence Erlbaum Associates, Inc.

Wegerif, R. (2006). A dialogic understanding of the relationship between CSCL and teaching thinking skills. International Journal of Computer-Supported Collaborative Learning, 1(1), 143-157.

Zemel, A., \& Koschmann, T. (2013). Recalibrating reference within a dual-space interaction environment. International Journal of Computer-Supported Collaborative Learning, 8(1), 65-87.

Zhao, K., \& Chan, C. K. K. (2014). Fostering collective and individual learning through knowledge building. International Journal of Computer-Supported Collaborative Learning, 9(1), 63-95. 